\title{
New sensors from old
}

A serendipitous discovery reveals that an existing fluorescent protein is actually a specific sensor for superoxide.

It is always nice to find a new use for something old, particularly when the new use is unique and valuable. It is even better when it provides an exciting biological discovery. This is precisely what happened to the authors of a recent paper in Cell.

Linda Groom and Robert Dirksen at the University of Rochester were trying to study calcium signaling in the mitochondria of muscle cells using the calcium sensor pericam but saw something they did not understand. This 'something' was unexpected random 10-20-second flashes in the matrix of single mitochondria expressing pericam (Fig. 1). They contacted Heping 'Peace' Cheng, discoverer of calcium sparks, and the two groups embarked on a collaboration to determine the origin of the flashes.

Cheng and his postdoc Wang Wang at the US National Institutes of Health initially interpreted the flashes as calcium 'marks' triggered by calcium 'sparks', but the more they looked, the more interesting it became. Cheng says: "We took one year to prove that the flashes were not calcium signals." In fact, the sensor still showed the flashes even after the scientists removed the entire calciumsensing domain, leaving only the circularly permuted YFP (cpYFP). So whatever was causing the flashes was acting on the fluorescent protein itself.

But if the flashes were not calcium signals, what were they? "First we thought it was going to be $\mathrm{pH}$ or voltage," says Dirksen. Fluorescent proteins are known to be affected by $\mathrm{pH}$, but when the researchers used EYFP instead, no flashes appeared. Finally after half a year they linked the flashes to redox changes, but it was still unclear what redox change or species cpYFP was detecting. "The first thing we suspected was that it was general redox potential," says Wang. Finally, after another half a year of work, during which time Cheng moved back to Beijing, they linked the signals to superoxide.

Dirksen says: "The demonstration that the sensor is detecting superoxide was very exciting because there are no existing probes that are reversible and specific for superoxide. Not only did we have this really exciting sensor for superoxide in the mitochondria,

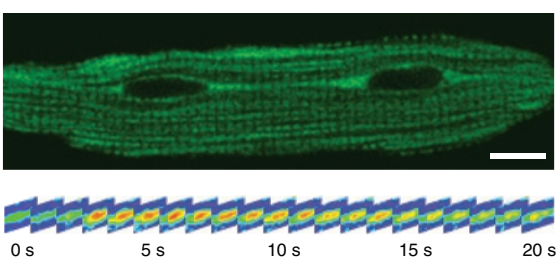

Figure 1 | Visualization of superoxide flashes in cardiac cell mitochondria using a fluorescent sensor for superoxide. Fluorescence micrograph showing the expression of the mitochondriatargeted superoxide sensor mt-cpYFP in a cardiac cell (top). Scale bar, $10 \mu \mathrm{m}$. Time-lapse imaging of a superoxide flash in a single mitochondrion (bottom). Image courtesy of W. Zhang, K. Li, $\mathrm{H}$. Fang and $\mathrm{H}$. Cheng.

but we were seeing these very beautiful bright transient events that nobody was ever aware of." The discovery of this phenomenon also launched the authors into the midst of a controversy. The mechanism and role of superoxide production in mitochondria turns out to be quite contentious. Their cpYFP results showed that the flashes are caused by transient openings of the mitochondrial permeability transition pore and are dependent on the activity of the electron transport chain, directly impacting existing theories of superoxide formation.

The mechanism by which the sensor is specific for superoxide and not hydrogen peroxide or other reactive oxygen species remains a mystery. Cheng believes that if someone could figure this out it might be possible to synthesize new types of superoxide indicators with better properties.

The $\mathrm{pH}$ sensitivity of cpYFP currently limits its use to the mitochondrial matrix. But as mitochondria are the primary source of superoxide in cells, the sensor is still very useful and provides a new and powerful tool for examination of the processes by which highly toxic superoxide is produced and regulated. According to Dirksen, both groups intend to continue following this exciting story: "Peace is going to continue on in the cardiac field, and I am going to continue in the skeletal muscle field." No doubt others will quickly follow.

\section{Daniel Evanko}

RESEARCH PAPERS

Wang, W. et al. Superoxide flashes in single mitochondria. Cell 134, 279-290 (2008). 